Article

\title{
Blockchain Enhanced Emission Trading Framework in Fashion Apparel Manufacturing Industry
}

\author{
Bailu Fu ${ }^{1}$, Zhan Shu ${ }^{2, *}$ (D) and Xiaogang Liu ${ }^{1}$ \\ 1 Fashion and Design College, Donghua University, Shanghai 200051, China; 1129106@mail.dhu.edu.cn (B.F.); \\ liuxg@dhu.edu.cn (X.L.) \\ 2 Department of Structural Engineering, Tongji University, Shanghai 200092, China \\ * Correspondence: shuzhan@tongji.edu.cn; Tel.: +86-21-6598-1582
}

Received: 19 March 2018; Accepted: 4 April 2018; Published: 7 April 2018

\begin{abstract}
Motivated by the recent blockchain technology originally built for bitcoin transactions, various industries are exploring the opportunities to redefine their existing operational systems. In this study, an innovative environmentally sustainable solution is proposed for the fashion apparel manufacturing industry (FAMI), which is energized by blockchain. Incorporating the Emission Trading Scheme (ETS), and a novel "emission link" system, the proposed framework exposes carbon emission to the public and establishes a feature to reduce the emissions for all key steps of clothing making. Fully compatible with Industry 4.0, blockchain provides decentralization, transparency, automation, and immutability characteristics to the proposed framework. Specifically, the blockchain supported ETS framework, the carbon emissions of clothing manufacturing life cycle, and the emission link powered procedures are introduced in detail. A case study is provided to demonstrate the carbon emission evaluation procedure. Finally, a multi-criteria evaluation is performed to demonstrate the benefits and drawbacks of the proposed system.
\end{abstract}

Keywords: blockchain; sustainability; fashion apparel industry; carbon trading; energy economics; Industry 4.0

\section{Introduction}

With the high degree of process automation and digitization brought by the fourth industrial revolution (Industry 4.0) [1,2], new possibilities to achieve more flexible and efficient productions and services have emerged [3,4]. Smart device computing, cloud computing, big data, Internet of Things (IoT), and Cyber-Physical Systems (CPS) are the key technologies of Industry 4.0 (e.g., [2,5,6]). Specifically, CPSs can monitor physical processes, create virtual copies of the physical world and make decentralized decisions. IoT is a dynamic network where physical and virtual entities have identities and attributes and use intelligent interfaces. For the machine-to-machine transactions involved in Industry 4.0, a reliable and secure managing system is a vital requirement to encounter the complexity of the new paradigms [7].

Blockchain technology provides decentralization, transparency, and immutability, which could offer a powerful fundamental system behind Industry 4.0 [8]. Simply put, the blockchain could be considered as a distributed ledger that provides a way for information to be recorded and shared by a community. In this community, each member maintains his or her own copy of the information and all members must validate any updates collectively through complex algorithms and cryptology replaces third-party intermediaries as the keeper of trust. With such decentralized peer-to-peer (P2P) features, the blockchain is regarded as a suitable system supporting Industry 4.0 [9]. Various organizations have begun exploring ways that blockchain might profoundly transform some of their most basic operations, from the way they execute contracts and carry out transactions to the ways they improve 
efficiency, engage potential customers, and more. New techniques are also emerging such as the Qtum service, which bridges the gap between blockchain networks and businesses [10].

The prosperity of blockchain technology also has great significance for the development of environmental sustainability, with the long-term goal to reduce the effects of climate change. However, the current applications of the technology in environmental sustainability are still in the primary stage. The existing environmental sustainability solutions are not open to the public. The collected data are less monitored and therefore might be falsified, leading to a series of problems such as serious data island effect and the lack of trust. Blockchain supported systems are designed to cure these problems. For example, in China, the Energy Blockchain Labs (founded in 2016) aims to provide financial services to the environmental protection industry based on blockchain technology [11]. In addition, a project called EP-Chain is now focusing on the development of blockchain, IoT, and fundamental level environmental protective (EP) applications [12]. The EP-Chain being built could store various EP data from different sources such as the environmental monitoring stations, devices, environmental purification products, and certified individuals. With such features, the EP-Chain aims at providing a secure, trustable system for data calculation, transformation, storage, and management.

Meanwhile, Khaqqi et al. [9] proposed a novel Emission Trading Scheme (ETS) model, also under the framework of Industry 4.0. The proposed ETS model uses blockchain technology and smart devices to improve compliance measure of ETS policy. With the transparency and immutability brought by blockchain, the accuracy of data essential for the scheme can be ensured. Thus, the functionality, consistency, credibility, and efficiency of the scheme can be improved. Furthermore, their study proposed a reputation-based trading system, which ultimately encourages the participants to adopt a long-term solution in emission abatement.

Apparel and fashion products, from fast fashion to luxury goods, are manufactured, stored, and distributed in global supply chains. Along with textiles, the annual turnover of the fashion apparel industry was estimated at 3 trillion USD in 2011 [13]. The rapidly changing world of fashion pushes toward overconsumption of resources such as water, grown cotton, and energy [14,15]. At the same time, this industry utilizes extensive amounts of chemicals. Unfortunately, current ETS is usually not designed for this industry. Hence, there exists an immense opportunity in this sector to contribute to positive change in terms of sustainability.

Focusing on the blockchain aided sustainable solutions, this study provides a blockchain supported environmentally sustainable solution for the fashion apparel manufacturing industry (referred to as FAMI hereafter). As the proposed system is energized by the blockchain, the study first highlights the blockchain technology in Section 2. Existing applications of the blockchain in the field of clean energy applications are also provided. Then, in Section 3, the blockchain aided ETS concept is proposed for FAMI. Instead of introducing a price control measure, it introduces a new approach to encourage the adoption of more sustainable and long-term solutions. Specifically, a systematic emission reducing standard for each product is encoded to the blockchain throughout an enhanced "emission link" system. The proposed framework has a "lead by the government, administrated by everyone" scheme. Furthermore, in Section 4, a case study to evaluate the carbon emission of a prototype product from the emission link is provided and the uncertainties are considered. In Section 5, a multi-criteria analysis of the established ETS framework is performed. Finally, a brief discussion of the proposed system is presented in Section 6.

\section{Blockchain and Current Applications to Improve Sustainability}

\subsection{Blockchain Technology}

The early blockchain concept was proposed by a developer hiding behind the pseudonym Satoshi Nakamoto [16], which has been fully validated through the bitcoin system implementations since 2009. Over the recent years, blockchain technology was further regarded as the fifth disruptive computing paradigm ever after the mainframe and personal computer paradigms, the Internet, and mobile and 
social networking [17]. Therefore, the blockchain is currently a widely studied research topic and is also being unleashed to multiple industries around the world that might revolutionary topple their existing infrastructures.

The blockchain entries could represent transactions, contracts, assets, identities, or practically anything else that can be digitally expressed using smart devices. New versions of blockchain technology implementation offer support for the implementation of smart contracts encoded in ledger's blocks [18], which implements different business rules that need to be verified and agreed upon by all peer nodes from the network. When a transaction arrives, each node updates its state based on the results obtained after running the smart contract. Such replication process offers a great potential for control decentralization.

Meanwhile, the integrity of the whole information is kept by all nodes within the blockchain network. The new edits and/or entries are initiated, validated, recorded, and distributed following the consensus algorithms that rely on the Proof protocols [19]. The more nodes joining blockchain, the more backups are made, providing reliability to the system. Therefore, all the recorded information is permanent, transparent, and searchable, which makes the entire transaction histories available to all community members. With such system established, a node could easily join or leave the blockchain network, securing the stability of the system. Besides, given a sufficient number of participants, the finished transactions could never be modified upon the public distributed ledgers.

The ownership and security of the transactions on the blockchain are provided by asymmetric cryptographic algorithms where the public keys are paired with the private keys. Blockchain participants use their private key (usually placed at the end of the data) to sign their own transactions, which are addressable on the blockchain network via public keys [20]. Mathematical functions are developed to compute the public keys from the data, but not the private keys. Therefore, the information is encrypted with private keys from the participants and could be publicly verified by solving the public keys [21].

More details and definitions of terms characteristic to the area of blockchain technology and crypto-currencies could be found in the existing work (e.g. [8,22]). In addition, some of the deficiencies of blockchain trading such as information leakage has been studied [23].

\subsection{Blockchain Applications to Improve Sustainability, an Example in the Clean Energy Industry}

Parallel to the newer solutions of power transmission considering environmental benefits (e.g., [24]), the blockchain in the field of energy is now reaching multiple markets over the world, with an aim to create a decentralized autonomous energy (DAE) community [22]. For example, decentralized solutions using blockchain peer-to-peer systems for managing demand response programs were proposed to improve current energy grid operations (e.g., [25,26]). The same method was applied to promote clean energy such as solar photovoltaic (PV) in China [27].

Besides, one could also refer to the start-up TransActive Grid [28] in Brooklyn, New York, which was co-founded in 2016 by the traditional micro-grid company LO3Energy and the blockchain technology company ConsenSys. With blockchain technology, TransActive Grid allows residents with solar PV panels to sell energy to their neighbors without a middle-party. The transactions are documented in the blockchain with very minimal need of human efforts, removing the possibility of corrupt or missing data [29]. Meanwhile, blockchain supported energy systems such as SolarCoin [30], Grid Singularity [31] and Grid+ [32] are developed and implemented. The economic performance of P2P energy sharing models were discussed [33].

During the 2016 EMART energy conference held in Amsterdam, Netherlands, 23 energy companies created an alliance to develop and use energy blockchain based on the transaction tool "Enerchain" developed by PONTON [34]. The encrypted order forms could be sent to the blockchain anonymously by a party. Other energy parties could directly view the order without the need of going through a centralized electricity company. In 2017, the Innogy SE Company, which is a subsidiary company of German energy group RWE, proposed a blockchain platform called Share\&Charge [35]. 
The operation of the developed blockchain platform was processed by the Ethereum technologies [36]. Thousands of blockchain based electric vehicle charging stations have been built. The Share\&Charge platform allows an individual to profit by sharing his or her charging station with others. Blockchain technology is expected to reduce the operational cost of Innogy SE. Later, the start-up Oxygen Initiative [37] joined Share\&Charge that transplanted the concept to serve the American drivers.

The decentralized grid distribution could improve the efficiency of clean energy, reducing the waste and broadening the markets. In China, for example, the company "Energo" was founded in Shanghai in 2016, which aims at using a similar concept to popularize clean and sustainable energy [38]. Blockchain is important in such applications so that transactions, such as producing or consuming energy, cannot be modified and are thus secure. Energo provides solutions that use microgrids and potentially allow any energy producers to sell energy to energy consumers. Energo's core products are based on the Qtum blockchain platform and include a hardware infrastructure under construction. Including the smart electricity meters, the distributed microgrid batteries, and the blockchain platform itself, the complete blockchain hardware system is used for measurements, transactions, and the settlement of clean energy. Designed for the distributed power producers, the smart electricity meter can be connected to the Energo app to buy and sell clean energy.

Potentially, the blockchain supported clean energy market is huge in China. Take the wind power system as an example, most wind farms are lumped in the "three north" area, i.e., northwest, northeast and north China where plain and mountainous landforms provide abundant wind resources. Currently, wind energy providers are selling the generated electricity to the state grid company. When wind speeds are fast, some of the generated power in the "three north" area is wasted due to the limit of power transmission network. Meanwhile, Shanghai, which is a mega city located relatively to the south, has to spend more than 20 Billion Yuan annually to improve its power capacities over the recent years. By building a complete microgrid parallel to the state grid and utilizing the blockchain technology, the wind energy could be better distributed and utilized, which will increase the profit and decrease the cost for all the related parties.

\section{Improvement of Environmental Sustainability in Fashion Apparel Manufacturing Industry}

Carbon emission is an important criterion to evaluate the product manufacturing process. Since the "Kyoto Protocol" came into effect [39], the carbon emission trading market has become a commodity trading market with great potential. While the social and economic effects of the ETS are topics still being discussed [40-42], multiple ETS have been emerging around the world. The European Union Emissions Trading System, also known as the European Union Emissions Trading Scheme (EU ETS), is the first large greenhouse gas emissions trading scheme in the world [43]. With its ETS established in December 2017, China is incubating one of the world's largest markets in the environmental protection industry. Announced at the 2009 United Nations Copenhagen Climate Change Conference, China is aiming at a short-term target to reduce $\mathrm{CO}_{2}$ emission by $40-45 \%$ by 2020 .

The fashion apparel industrial sector is a primary source of greenhouse gas (GHG) emissions, including $\mathrm{CO}_{2}$, one of the principal sources of global warming. The traditional FAMI is notorious for utilizing extensive amounts of natural resources including water, grown cotton, energy, and chemicals. Noted by CleanMetrics [44], clothing and accessories are the consumer goods that, next to food and beverages, are purchased most often and also replaced most frequently. Besides, according to WWF report [45], because of the scope of the industrial sector's activities, it is a significant GHG emitter with apparel and textiles accounting for about $10 \%$ of the total carbon emissions, and with textiles being the fifth largest contributor to $\mathrm{CO}_{2}$ emissions in the USA. Farms producing materials such as cotton and silk could also bring significant pollutants [14,46], as can retail operations, involving excessive packaging and employing environmentally unfriendly electricity power supplies [15,47].

Some nations are now discussing to place the FAMI as one of the focal industries for further exploration of the respective green manufacturing-related policies, and various measures are proposed [48]. The concept of carbon footprint [49,50] and the carbon footprint tax [51] has been 
proposed, applied and discussed. In the UK, for example, the carbon footprints for various clothing have been summarized [52]. Other regulatory policies including Mandatory Cap, Cap\&Trade, and Carbon offset have been proposed and applied around the world [53]. Researchers also provided operations strategies and tactics to reduce pollutant emissions (e.g., [54-56]). In addition, companies such as Adidas, H\&M, Marks and Spencer, and Nike provide annual sustainability reports from their official websites to address the issue [57-60]. However, more efforts are needed to make this sector environmentally friendly.

Under the framework of Industry 4.0, this section constructs a simple and intuitive blockchain supported ETS framework with emission link in the FAMI (referred to as BCELF hereafter). The blockchain technology, specifically, has proven to be a viable solution also compatible with Industry 4.0 [9]. Compared with solitary efforts from government authorities advocating sustainable policies, the blockchain could provide a platform that pushes forward the topic to the public with transparent and trustable records [9,61]. With the proposed framework, more details of FAMI are included in the ETS framework. Specifically, the BCELF, the clothing manufacturing life cycle, and the emission link powered procedures are introduced in detail.

\subsection{Blockchain Supported Emission Trading Scheme}

Most current ETS systems are not designed for the FAMI. Meanwhile, there are only finite amount of existing studies on the blockchain supported ETS [9,12,61]. Blockchain secures creditable entries, which will virtually increase the trust between different participants. Every step is open, transparent, and immune to falsifications. All the distribution, transfer, cancellation and inventory of the carbon allowance will be kept and managed irreversibly. Moreover, current studies have shown that the credible information provided by blockchain could protect the system from fraud and other problems such as double counting issues $[9,41]$.

The proposed BCELF does not fundamentally change the conventional schemes, rather, it enhances them by incorporating more details based on the manufacturing procedures of the products. With the implementation of BCELF, the carbon emissions of the industry are exposed to the public and a feature to reduce the emissions throughout all key manufacturing steps is established. Specifically, as shown in Figure 1, there are four entities in the proposed system, i.e., the authority, the auditor, the firms, and the individuals. Each entity has different issuances and responsibilities, which are presented in Table 1.

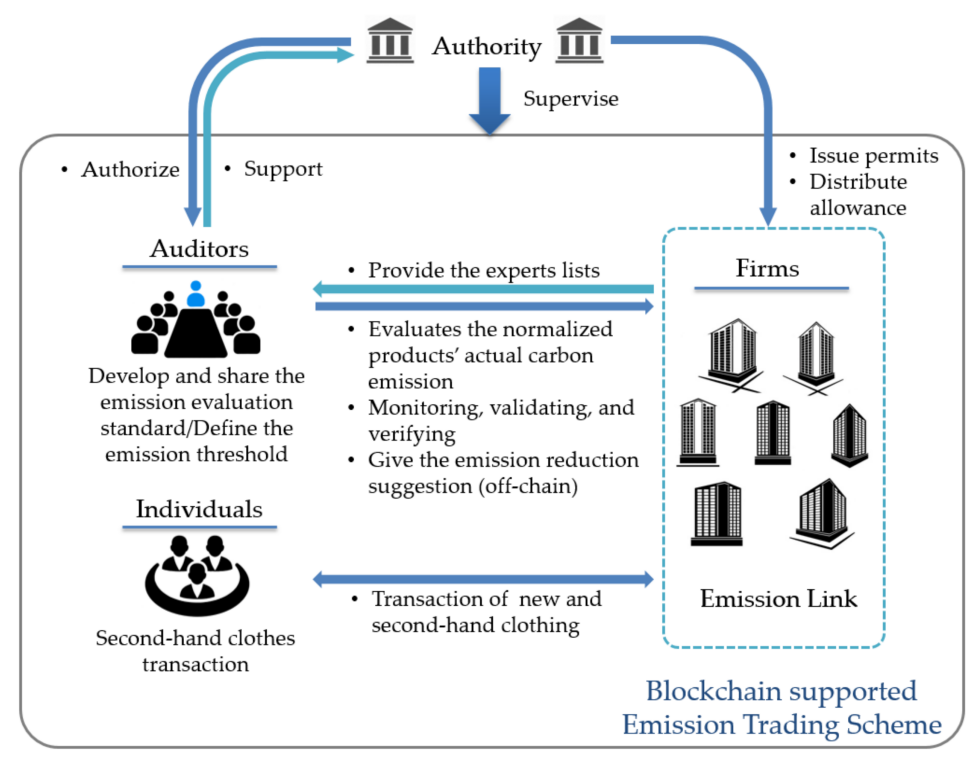

Figure 1. Blockchain supported emission trading scheme. 
Table 1. Description of entities and their functions in the BCELF system.

\begin{tabular}{|c|c|c|c|c|c|}
\hline \multirow{2}{*}{ Role } & \multirow{2}{*}{ Holder } & \multicolumn{3}{|c|}{ Capacity } & \multirow{2}{*}{ Responsibility } \\
\hline & & & Issuance & Transaction & \\
\hline Authority & $\begin{array}{l}\text { Ministry of textile } \\
\text { industry or } \\
\text { the equivalent } \\
\text { government body }\end{array}$ & \multicolumn{2}{|c|}{ Issues the permits (allowance units) } & $\begin{array}{l}\text { Distribute carbon } \\
\text { allowance units }\end{array}$ & $\begin{array}{l}\text { 1. Create, explain and } \\
\text { enforce the ETS policy } \\
\text { 2. Facilitate the } \\
\text { implementation of the } \\
\text { blockchain supported } \\
\text { carbon ETS }\end{array}$ \\
\hline \multirow{4}{*}{ Auditors } & \multirow{4}{*}{$\begin{array}{l}\text { Government divisions, } \\
\text { experienced consulting } \\
\text { firms, universities, etc. }\end{array}$} & \multirow[b]{2}{*}{ On-chain } & \multirow{2}{*}{$\begin{array}{l}\text { 1. Carbon emission } \\
\text { standards of the products } \\
\text { 2. Carbon emission } \\
\text { threshold of the } \\
\text { Emission Link }\end{array}$} & \multirow[b]{2}{*}{-} & \multirow{2}{*}{$\begin{array}{l}\text { 1. Summarize and } \\
\text { organize the data from } \\
\text { the blockchain } \\
\text { 2. Monitoring, validating, } \\
\text { and verifying }\end{array}$} \\
\hline & & & & & \\
\hline & & \multirow[b]{2}{*}{ Off-chain } & \multirow[t]{2}{*}{ 1. Reports to the authority } & \multirow[b]{2}{*}{ - } & \multirow{2}{*}{$\begin{array}{l}\text { 1. Analysis and report } \\
\text { industry/firms/products } \\
\text { actual carbon emission } \\
\text { 2. Provide customized } \\
\text { carbon emission } \\
\text { reduction strategies }\end{array}$} \\
\hline & & & & & \\
\hline Firms & $\begin{array}{l}\text { Business institutions in } \\
\text { textile industry }\end{array}$ & \multicolumn{2}{|l|}{ - } & $\begin{array}{l}\text { Send or receive carbon } \\
\text { allowance units as } \\
\text { seller or buyer }\end{array}$ & $\begin{array}{l}\text { 1. Report emissions } \\
\text { 2. Comply with } \\
\text { the blockchain } \\
\text { 3. supported ETS rules } \\
\text { including the } \\
\text { Emission Link }\end{array}$ \\
\hline Individuals & Customers & \multicolumn{2}{|l|}{ - } & $\begin{array}{l}\text { Send or receive carbon } \\
\text { allowance units mainly } \\
\text { as seller }\end{array}$ & $\begin{array}{l}\text { 1. Recycling the used } \\
\text { clothing to firms } \\
\text { or individuals }\end{array}$ \\
\hline
\end{tabular}

The authority is the initiator and the supervisor of the blockchain project in FAMI. A typical authority could be the ministry of textile industry or the equivalent government body. As such project is usually led by the authority, a few key responsibilities should be fulfilled by this crucial entity. First, the authority designs, explains, and enforces the ETS policy. The design of the ETS policy should consider elements such as the scope of the policy, the cap allocation, the allowances or permits distribution, the offset policy, the trading mechanism, the ETS period design, the Monitoring, Reporting and Verification (MRV) procedure, and the expansion potential [62]. Second, the authority issues the permits and distributes the carbon allowances to all products. For the carbon allowance unit, one could refer to the EU Allowance Unit of one tonne of $\mathrm{CO}_{2}$, or "EUA", or any units "fungible" with the equivalent "Assigned Amount Unit" (AAU) of $\mathrm{CO}_{2}$ defined under Kyoto Protocol [39]. Thirdly, the authority should facilitate the implementation of the blockchain supported carbon ETS.

With the mandatory requests from the authority, the firms are enforced to join the system. Within the BCELF, a firm-to-firm carbon "emission link" is established parallel to the transactions of their products. The firms could trade the carbon emission units from the blockchain. The details of the evaluation and transaction process will be introduced and discussed in the subsequent sections.

Designated by the authority, the auditors are groups of independent committees in the blockchain that constitute a distributed network of "think tanks" that link the authority and ETS system. The duties of auditors can be carried out by the government divisions, universities, or experienced consulting firms. The detailed deliverables of the auditors include two parts, i.e., the "On-chain" evaluations and "Off-chain" reports. First, the auditors establish the carbon emission standards of all products from all the firms with the "emission link" system from the blockchain. For each product, the evaluation criteria are standardized with a collaborated effort from a group of experts specialized in the manufacturing process and the environmental protection fields. The criteria are systematic and compatible with the blockchain such that the experts only need to establish and update the criteria. In addition, emission monitors are installed for the key manufacturing steps to collect the emitted waste. The result of carbon emission evaluation of a product line could be updated on the blockchain once a more environmentally friendly solution is adopted. Meanwhile, the operation of the emission link requires less human efforts with the automation brought by blockchain. Second, different from the "On-chain" evaluations, the auditors offer "Off-chain" services to summarize and to organize the data from the blockchain. 
Such service provides a solid support for the authority to supervise and to improve the operations of the ETS. Such "Off-chain" service could include, but is not limited to, the determination of carbon emission allowances on the product permits, carbon emission threshold, and other concerns that help the authority to logically balance, manage and control the ETS. The auditors could also offer consulting services for firms to develop optimized strategies toward energy savings and emission reductions.

Within the blockchain, the individuals could also be involved in the whole life cycle of the clothing manufacturing. The individuals can easily track the clothing carbon emissions from the blockchain, which help to support their consuming decisions. Meanwhile, the blockchain provides a link between the firms and the individuals such that more efficient clothing recycling could be achieved. The individuals have the option to sell second-hand clothing to other individuals, available fashion brands recycling projects, or recycling firms. Carbon emission allowances or other benefits could be rewarded as the incentives to the individuals throughout such efforts.

\subsection{Carbon Emission from Fashion Apparel Manufacturing Chain}

The fashion apparel industry manufacturing chain is relatively long. The proposed blockchain supported ETS is a more sustainable solution where the firms are enforced to operate, choose facilities, and manufacture products following the environmentally friendly approaches. The FAMI generally contains three major phases [63], i.e., producing raw materials, processing materials, and making clothes. As shown in Figure 2, Phase 1 includes natural fiber production from animals/plants and man-made fiber production from a chemical industry. Phase 2 includes spinning; fabric production (including warping, slashing, weaving, and knitting procedures); wet treatment (including preparation, dyeing, finishing, and printing procedures). Phase 2 provides the ready-to-use materials for Phase 3 , where the materials are cut, sewed, and further treated into clothes. In particular, dyeing and finishing procedures are the most polluting procedures, producing great amount of waste water and carbon emission.

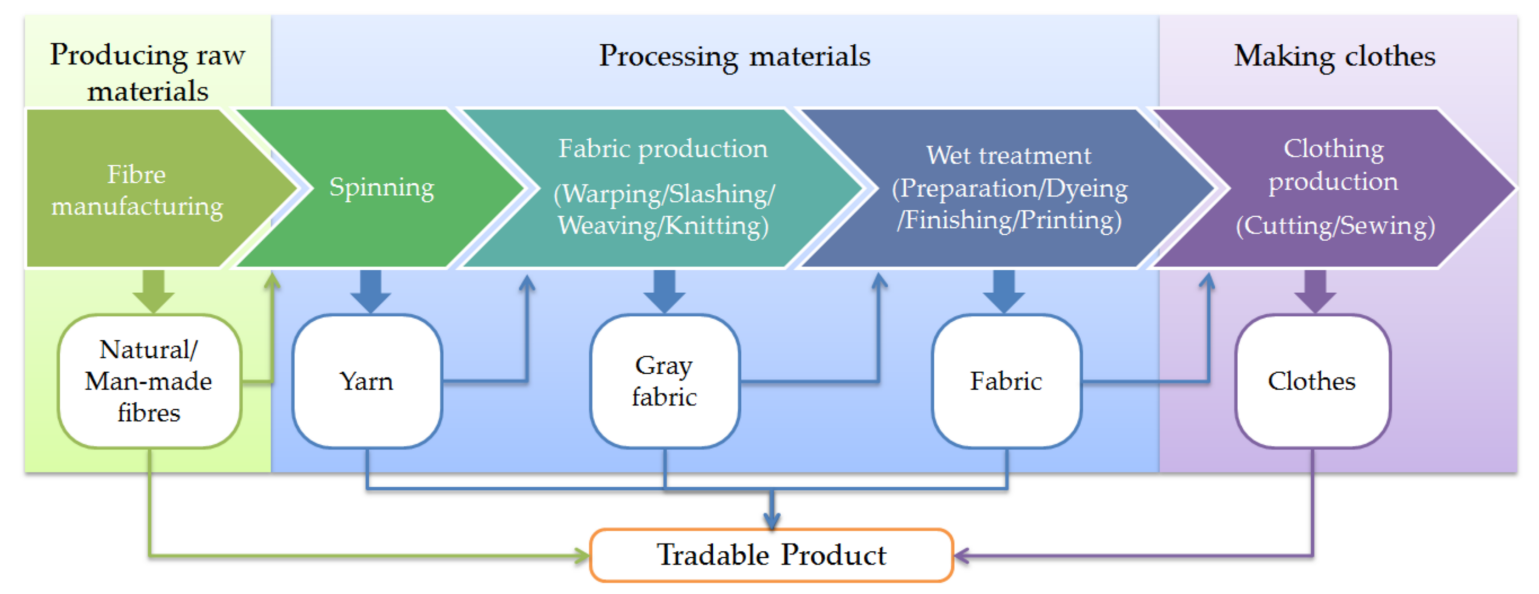

Figure 2. Three major phases and their tradable products for the apparel manufacturing industry.

Together with the establishment of carbon emission standards, the environmentally friendly guidance is also provided for each of the three phases. Phase 1 is the raw materials production. Different choices of raw materials have large impacts in the following procedures such as dyeing methods, consumer-care requirement. In fact, lots of natural fibers such as cotton are not as eco-friendly as people believe. Water, fertilizers, herbicides and pesticides are used during the cotton planting. Using alternative fibers such as organic hemp or recycled cotton to replace natural fibers or synthetics can significantly reduce the carbon emissions.

For Phase 2, a firm could find cleaner energy resources and manage the wastewater with more sustainable approaches. More sustainable dyes are suggested such as vegetable dyes. For Phase 3, 
a firm could try cleaner production processes, improve the production efficiency for shell fabrics, and adopt other methods that consume less energy and/or produce fewer wastes. Furthermore, over-packaging should be avoided. Instead, no package or using recycled coat hangers could be considered. Notice that the proposed model is just an example focusing on the manufacturing stage. The follow-up stages should also be improved, e.g. to avoid the long-distance distributions for the supply chain [64] or choose the lower carbon emission way. Furthermore, as shown in the white boxes in Figure 2, the tradable products are provided for each of the corresponding steps. The proposed system builds a carbon emission link for each product and records the emissions impartially on the public ledger provided by the blockchain.

Notice that the aforementioned carbon emission evaluation standards for each product are the prerequisites of the proposed system. With the collaborated efforts from multidiscipline experts, the standards evaluate the carbon emissions for each product considering four aspects, i.e., supply, energy and fuel, waste, and labor (Figure 3).

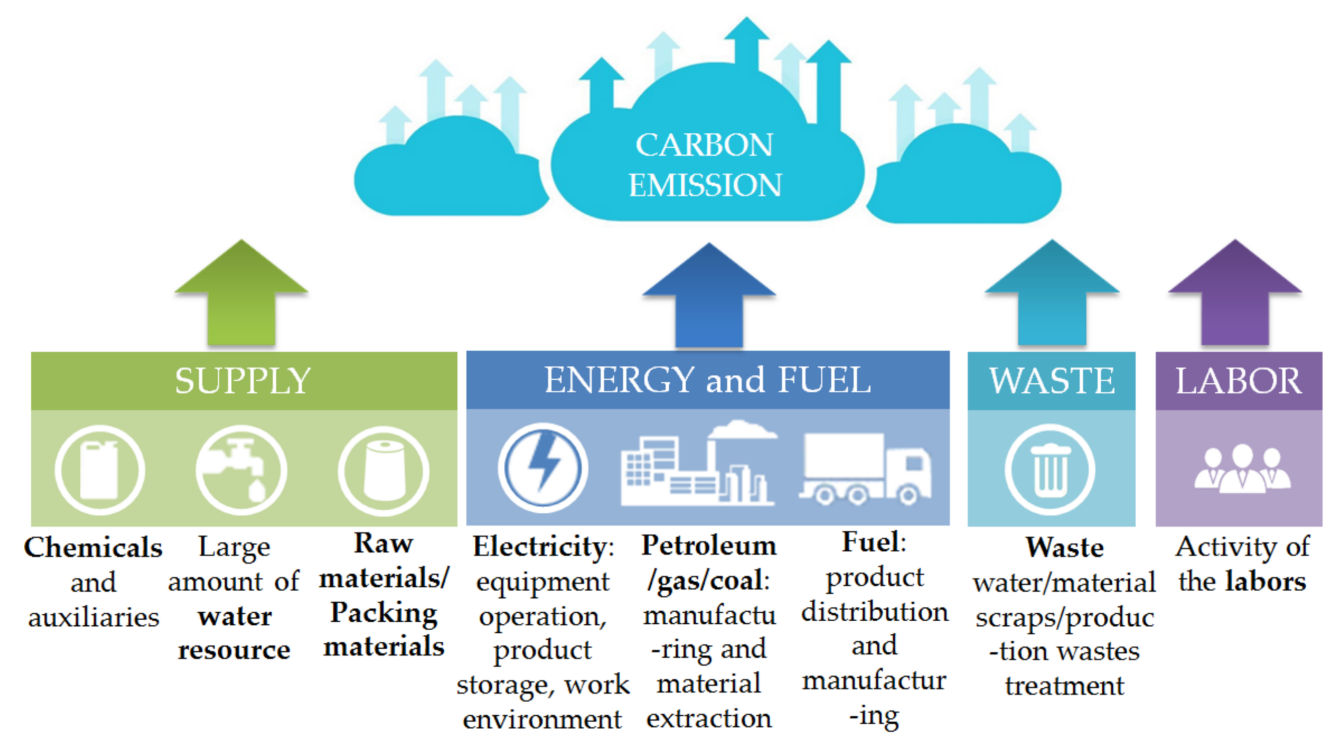

Figure 3. Four major points for the evaluation of each product of the blockchain.

The expert team should first evaluate the supply part, which includes the equivalent carbon emissions from chemicals and auxiliaries, the amount of water resource, raw material/packing material, etc. Taking the raw materials choice as an example, environment benchmarks of textile fibers are shown in Figure 4 [65], which are proposed according to four aspects: non-polluting to obtain, process and fabricate; made from renewable resources; fully biodegradable; and reusable/recyclable. Besides, carbon emission evaluation functions should be provided for the sizing agent and auxiliaries used in weaving procedure, the chemical dyeing and auxiliaries used in dyeing procedure, specific chemical reagents used in the finishing procedures of various fabrics, the large amount of water resource consumed during the spinning and weaving procedures, etc. For example, the carbon emission related to water resources was investigated $[66,67]$. 


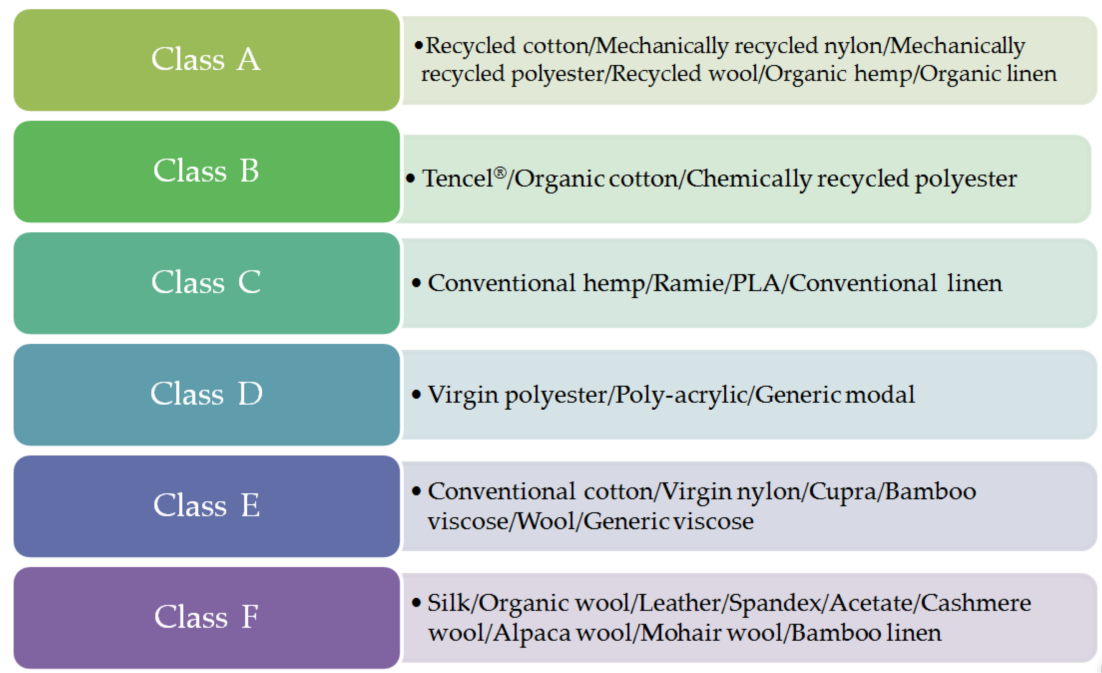

Figure 4. Environment benchmarks of textile fibers.

The second point of evaluation focuses on the energy and fuel consumptions. For example, electricity is consumed for equipment operation, product storage, and work environment such as the lighting, air-condition, ventilation, etc. Non-renewable energy such as petroleum is often used for polyester and nylon fiber extraction. In addition, non-renewable energy is also used for specific manufacturing procedures. Fuel is used for the procedures related to the manufacturing, heating and product distributions. Machines or smart devices should be installed to facilitate the measurement of the consumed energy. Consequently, the carbon emission while generating the consumed amount of energy and fuel could be evaluated by the functions developed according to the existing studies [68] or by the expert teams.

Thirdly, the wastes should be evaluated for the product, which refers to the wastewater, material scraps, and production wastes. One could refer to the existing studies to develop the carbon emission evaluation functions for the waste treatments [69]. In addition to the excessive wastewater and material in each procedure, significant production wastes should be considered such as the fiber lint, yarn waste, and fabric scraps in the spinning and weaving procedures. Moreover, packaging waste could be addressed in several procedures. Finally, the evaluation should also consider the equivalent carbon emissions from the labors due to the labor-intensive nature of the textile industry (e.g., [70]).

\subsection{Emission Link}

The emission link for the FAMI is constructed on the blockchain for the ETS system. The emission link is an executer of the established carbon emission standards, which evaluates the carbon emission more systematically for the products of the FAMI. With the emission link, the firms are motivated to implement more sustainable strategies. The carbon emission is evaluated for a product considering the supply, the consumed energy and fuel, the generated waste, and the hired labor. If a firm wants to take actions to reduce carbon emission of a product, it could get the off-chain consulting services from the auditors, which cover more details involved in the manufacturing procedures. Besides, an evaluated product should be "normalized" in the sense that it shares the same functionality as other peer products. The normalization of a product before the emission link evaluation is important as it encourages the products with higher efficiencies.

Utilizing the measurement machines and algorithms rigorously guided by the established standards, the blockchain supported evaluation of the carbon emission requires less human effort and therefore is less likely to be corrupted. In addition, the emission monitoring devices are required to be installed for some key manufacturing steps. The one-time carbon emission evaluation, as well as the 
long-term monitored results, is recorded on the blockchain. When the emission of a certain product is evaluated, its carbon emission could be computed together with the blockchain transactions.

The established standards also provide a guide to reduce carbon emissions. To reduce the carbon emission of a product, a firm could improve both its manufacturing methods and the manufacturing procedures. Improved methods with less carbon emission could be realized by selecting the environmentally friendly materials and machines. The example in Figure 5 assumes an original production line includes three steps and each of the steps has multiple inputs (from the supply), i.e., input $_{1,1 \ldots 1, \mathrm{n}}$, $_{\text {nput }}, 1 \ldots 2, \mathrm{x}$, and input $\mathrm{t}_{3,1 \ldots 3, y}$. A more sustainable solution is to adopt a newer step, i.e., Step 4 with input $4,1 \ldots 4, z$, which produces less carbon output that replaces Steps 2 and 3 . On the other hand, with the production method fixed, the manufacturing procedures could also be improved to reduce carbon emissions. The firm could refer to the major points of emission mentioned in Figure 3 for improvements. Efforts could also be taken to reduce the carbon emission for each of the three production steps.

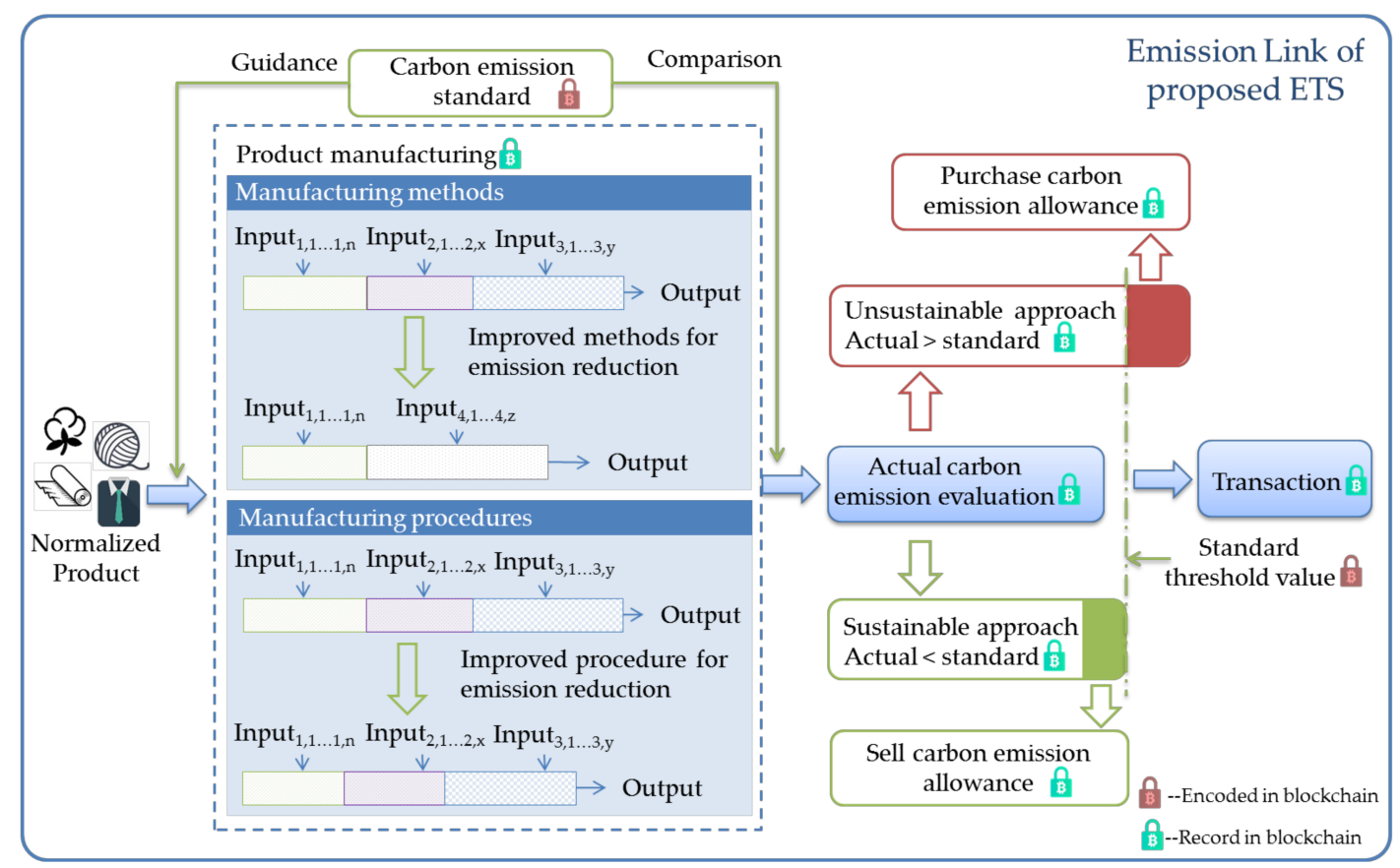

Figure 5. The emission link of the blockchain supported emission trading scheme.

Supported by the auditors, the developed blockchain, and the smart devices, the actual carbon emission for each normalized product is evaluated at the firm level. To better demonstrate the carbon emission process of the emission link, a case study is provided in the subsequent section. In addition, the uncertainties of the evaluation are also analyzed. The evaluated results are recorded on the blockchain, which is fully accessible to the public. The transparency and immutability features of the blockchain provide a reliable and more secure ETS system.

Then, the following steps are straightforward: if the actual emission of a product is higher than the standard defined threshold, the firm must purchase more carbon emission allowances from the market along with the transactions of the product. On the other hand, a firm benefits from an environmentally sustainable product through the bonus carbon emission allowances available through trading or auctions. Decided by the authority, the standard threshold starts high and usually decreases every year at the implantation stage of the ETS system and will converge to a more stable value after decades of operations. In addition, the determination of the carbon allowance price might be a challenge for the existing ETS systems. It is a complicated topic considering many issues such as the supply and demand of a market that needs a long-term plan from the authorities. However, with the proposed 
model, the auditors on the blockchain could provide solutions to this problem. The authority could refer to the existing approaches such as the EU ETS and the pilot ETS project in China. The price usually starts from relatively low and will increase and is being balanced by auctioning process.

\section{Case Study and Uncertainties}

\subsection{Case Study}

The carbon emission evaluation of a prototype product, Product A, is provided here as a case study. In Figure 6, three main procedures to manufacture the product require three different kinds of machines (i.e., $\mathrm{M}_{1}, \mathrm{M}_{2}$, and $\mathrm{M}_{3}$ ). Commonly used machines in FAMI include the picker machine, the carding machine, the combining machine, etc. The numbers of each kind of machine needed are $n_{1}$, $\mathrm{n}_{2}$, and $\mathrm{n}_{3}$, respectively. For the first procedure, three kinds of supply materials are required (i.e., $\mathrm{S}_{1}$, $\mathrm{S}_{2}$, and $\mathrm{S}_{3}$ ). In addition, assume that the machines in the first procedure consume two types of energy (i.e., $E_{1}$ and $\left.E_{2}\right)$ and only one kind of trained labor $\left(L_{1}\right.$ is the number of people involved in the first procedure) are needed. The outcome of the first procedure is a temporary product required for the next procedure, $\mathrm{P}_{1}$, and three kinds of wastes (i.e., $\mathrm{W}_{1}, \mathrm{~W}_{2}$, and $\mathrm{W}_{3}$ ). For the second step, the supply $\mathrm{S}_{1}$ is required. One kind of energy is consumed (i.e., $\mathrm{E}_{1}$ ), and two kinds of labors (number of people: $\mathrm{L}_{2}$ and $\mathrm{L}_{3}$ ) are needed. The outcome of the second procedure is a temporary product required for the last procedure, $\mathrm{P}_{2}$, and two kinds of wastes (i.e., $\mathrm{W}_{1}$ and $\mathrm{W}_{3}$ ). Finally, two more supplies, $\mathrm{S}_{4}$ and $\mathrm{S}_{5}$, are required for the last procedure. One kind of energy is consumed (i.e., $E_{1}$ ). One kind of labor with a total number of $\mathrm{L}_{4}$ people is needed. The outcome of the last procedure is Product $\mathrm{A}$, and one kind of waste (i.e., $\mathrm{W}_{4}$ ).

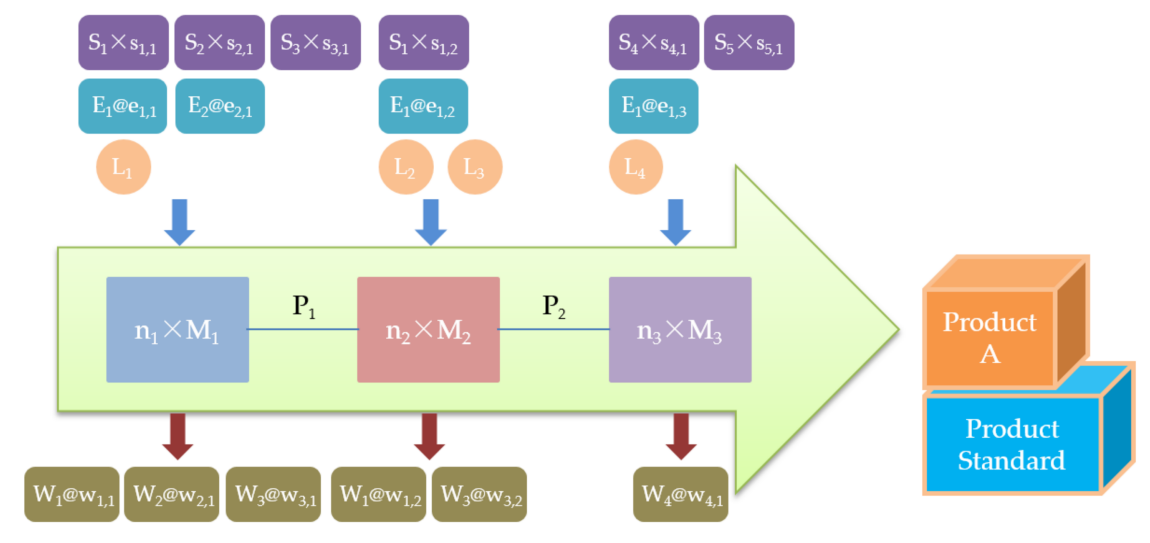

Figure 6. Manufacturing procedures of Product A.

The labor only considers the number of people operating the machines, while the supervisors and company managers are not included. The carbon emission is then computed with Equation (1):

$$
C E=\left[\sum_{i=1}^{i s} f s_{i}\left(\sum_{j=1}^{j s} s_{i, j}\right)+\sum_{i=1}^{i e} f e_{i}\left(\sum_{j=1}^{j e} e_{i, j}\right)+f l\left(\sum_{i=1}^{i l} L_{i}\right)+\sum_{i=1}^{i w} f w_{i}\left(\sum_{j=1}^{j w} w_{i, j}\right)\right] \times \phi
$$

where $C E$ stands for the total carbon emission; and $f s, f e, f l$, and $f w$ are the evaluation functions for the supply, energy, labor, and waste, respectively. These functions should be carefully studied and determined from the research institutions. In addition, is is the number of different supplies; $j s$ is the maximum use of a supply over different procedures; $s_{i, j}$ represents the amount of supply $i$ used in the $j$ th procedure; $i e$ is the number of different energies; $j e$ is the maximum use of a type of energy over different procedures; $e_{i, j}$ represents the amount of energy $i$ used in the $j$ th procedure; $i l$ is the total types of trained labors; $L_{i}$ is the number of trained person working in that category; $i w$ is the number of different wastes; $j w$ is the maximum use of a type of waste over different procedures; $w_{i, j}$ 
represents the amount of waste $i$ used in the $j$ th procedure; and $\phi$ is the modification factor for the product normalization.

For example, with the three steps of procedures, Product A is manufactured. The total carbon emission could be computed with Equation (2):

$$
C E=\left[\begin{array}{l}
f s_{1}\left(s_{1,1}+s_{1,2}\right)+f s_{2}\left(s_{2,1}\right)+f s_{3}\left(s_{3,1}\right)+f s_{4}\left(s_{4,1}\right)+f s_{5}\left(s_{5,1}\right) \\
+f e_{1}\left(e_{1,1}+e_{1,2}+e_{1,3}\right)+f e_{2}\left(e_{2,1}\right) \\
+f l\left(L_{1}+L_{2}+L_{3}+L_{4}\right) \\
+f w_{1}\left(w_{1,1}+w_{1,2}\right)+f w_{2}\left(w_{2,1}\right)+f w_{3}\left(w_{3,1}+w_{3,2}\right)+f w_{4}\left(w_{4,1}\right)
\end{array}\right] \times \phi_{A}
$$

In Equation (2), $\phi_{A}$ is computed by dividing the efficiency of Product A by the efficiency of the standard compatible product. In addition, uncertainties should be considered, as the estimation formula provides a bounded range of carbon emissions rather than a single value. Uncertainty analysis is provided in the Section 4.2.

\subsection{Determination of the Uncertainties}

Uncertainties should be addressed while evaluating the carbon emissions. The overall uncertainty of a product is the accumulated uncertainties from all the individual parameters. The propagation of uncertainty [71] is highlighted here for the carbon emission evaluations. The total uncertainty for $\mathrm{N}$ parallel uncertainties is estimated by Equation (3):

$$
U_{c}=\frac{\sqrt{\sum_{n=1}^{N}\left(U_{s n} \cdot e_{s n}\right)^{2}}}{\left|\sum_{n=1}^{N} e_{s n}\right|}
$$

where $U_{c}$ is the total uncertainty (unit: \%), $U_{s n}$ is the $n$th independent uncertainty (unit: \%), and $e_{s n}$ is the $n$th emission according to the evaluation. For example, two kinds of fuels are used to manufacture a unit of product. The $\mathrm{CO}_{2}$ emissions are $30 \pm 2 \%$ tonne and $40 \pm 10 \%$ tonne for the fuels, respectively. The total uncertainty could be calculated according to Equation (4):

$$
U_{c}=\frac{\sqrt{(30 \times 0.02)^{2}+(40 \times 0.1)^{2}}}{|30+40|}=5.78 \%
$$

In addition, when the uncertainties are in series, the total uncertainty is estimated by Equation (5):

$$
U_{c}=\sqrt{\sum_{n=1}^{N}\left(U_{s n}\right)^{2}}
$$

where $U_{c}$ is the total uncertainty (unit: \%) and $U_{s n}$ is the $n$th uncertainty in series (unit: \%). For example, the production of a unit of product burns $90 \pm 5 \%$ tonne lignite. The $\mathrm{CO}_{2}$ emission factor of the lignite is $2.1 \pm 10 \%$ tonne $\mathrm{CO}_{2}$ per Ton of lignite. Then, the total uncertainty is computed as Equation (6):

$$
U_{c}=\sqrt{(5 \%)^{2}+(10 \%)^{2}}=11.2 \%
$$

\section{Multi-Criteria Evaluation}

A multi-criteria evaluation method was proposed by Konidari and Mavrakis [72], which has been commonly used to evaluate of climate policies (among others, $[9,73,74]$ ). Such analysis compares the policy options against a plurality of environmental, economic and procedural criteria to identify options that would not only be feasible but also effective in overcoming present shortcomings and avoiding 
them in the future. This section evaluates the benefits against the drawbacks of the proposed system using the multi-criteria evaluation method. The evaluated grades for a certain criterion are divided into 11 levels, from null to excellent, which is originally provided by Konidari and Mavrakis [72] and modified by Khaqqi et al. [9]. The levels and their corresponding grades are summarized in Table 2.

Table 2. Grade scale for the assessment of the sub-criterion. Source: [72] modified by [9].

\begin{tabular}{cc}
\hline Assessment & Grade \\
\hline Null & $0.0-0.5$ \\
Slightly more than null, less than very bad & $0.5-1.5$ \\
Very bad & $1.5-2.5$ \\
Bad & $2.5-3.5$ \\
More than bad less than moderate & $3.5-4.5$ \\
Moderate & $4.5-5.5$ \\
More than moderate less than good & $5.5-6.5$ \\
Good & $6.5-7.5$ \\
More than good less than very good & $7.5-8.5$ \\
Very good & $8.5-9.5$ \\
Excellent & $9.5-10.0$ \\
\hline
\end{tabular}

Then, three ETS systems are analyzed, scored, and compared in this section, i.e., a conventional ETS system, the blockchain-enabled reputation-based ETS (BCRB) provided by Khaqqi et al. [9], and the BCELF. Besides, the conventional ETS in FAMI is also evaluated. The system from Khaqqi et al. [9] is selected because it is also a blockchain supported ETS system. Specifically, the ETS segments the market according to the reputation of a firm. Higher reputation allows the firms to access and choose from more trade offers. If such system could be well implemented, a long-term carbon emission abatement could be realized.

The study inherits the evaluation of the grades for the conventional ETS considering its status in different nations and incorporating various uncertainties $[9,72,73]$. Overall, the existing ETS systems are scored 6.69, considering their strengths and drawbacks. The ETS policies are also improving from the past experiences. In addition, the score of BCRB are referenced from Khaqqi et al. [9], i.e., a lower bound of 6.97 and a higher bound of 7.37. It is shown that considering all the criteria that determined the viability of a climate change policy, the benefit of the BCRB exceeds the drawback.

\subsection{Assessment of Environmental Performance}

According to Clò et al. [73], a conventional ETS provides GHG reduction throughout the limit/cap method, leading to a "good" grade with score 7. Due to the reputation system, the level could be improved to "more than good less than very good" with an average score of 8.15 . Without the specifications in the FAMI, a conventional ETS system only evaluates the energy consumption. The supply, waste, and labor, which are critical during the manufacturing process, are not evaluated. Therefore, the conventional ETS in FAMI only receives a moderate score of 5. The proposed ETS addresses all the carbon emissions from the systematically designed carbon emission standards. Upon its implementation, the carbon emission of the apparel manufacturing processes could be reduced comprehensively. Therefore, the proposed system gets a much higher score of between 8.2 and 9.1.

The indirect environmental effects are decided by the additional outcome of the mechanism. According to Khaqqi et al. [9], the criteria for conventional ETS and BCRB are measured using the same performance indicator, leading to the same score. Therefore, the grade for the indirect environmental effects is set equal to the direct contribution of the two systems. For BCELF, the involvement of individuals improves the indirect environmental social influences, leading to a slightly higher score. 


\subsection{Assessment of Political Acceptability}

The static cost efficiency sub-criterion is an important sub-criterion in the assessment of political acceptability [72]. For the BCELF, establishing the standard and encoding the carbon emission evaluation procedure to the blockchain only lead to limited financial burdens. Meanwhile, when the scheme is successfully established on the blockchain, much less human effort is required for the operation of the system, leading to a reduced cost. Therefore, the proposed framework has a slight advantage (i.e., lower bound at 7.2 and upper bound at 7.5) over the conventional ETS.

The second sub-criterion, dynamic cost efficiency, analyzes the self-improve capacity of a system. It is shown that the conventional ETS is usually less efficient compared with a blockchain supported ETS [75]. The blockchain supported ETS could quickly support research and any technical advancements to effectively reduce carbon emissions, which is considered very good (scoring 8.6-9).

The competitiveness sub-criterion evaluates the extent to which a policy option is likely to increase the short-run cost of production in the ETS sectors. The higher the carbon price is, the higher a firm's compliance cost is. The more details there are included in the policy, the higher is a firm's compliance cost. The conventional ETS in FAMI is currently less demanding than other industry sectors, which leads to a score of 6 . On the other hand, the BCRB as well as the proposed system get lower scores (i.e., 4.0-4.5 and 4.5-5.0) due to the adverse effects that increase the production costs.

The equity sub-criterion evaluates the fairness of the distribution of permits, compliance cost and reduction responsibility. For the proposed ETS, as the standards are developed and encoded to the evaluation system, a complete carbon emission could be achieved. For example, as labor is also considered in addition to the power consumption, the firms would not tend to use more labors to manually craft the product instead of using machines consuming energy. The established system is complete, fair, and therefore reduces the uncertainties. The score is evaluated between 8 and 8.5, which belongs to the level "More than good less than very good".

Flexibility is defined as the ability to provide different options on how participants may comply with the ETS regulations. Similar to the conventional ETS, the proposed scheme allows participants to choose any options to comply with the regulation. Therefore, the proposed system is scored close to that of the conventional ETS.

The last sub-criterion, stringency for non-compliance, addresses the mechanism's capacity to monitor the level of compliance and to impose sanctions on those who fail to comply. The blockchain supported system is much more superior to the conventional ETS due to the automation, transparency and immutability natures. Therefore, the proposed system is evaluated "very good" with a score between 8.5 and 9.5 .

\subsection{Assessment of Feasibility of Implementation}

To define how well an established scheme could be implemented, the first sub-criterion is implementation network capacity, which evaluates the effective capacity of the involved institutions to design, support and implement the chosen scheme. Specifically, the technological infrastructure, trained personnel, data credibility and information transparency could be used as assessing indicators. To implement a blockchain supported ETS, additional support is needed to construct the technological infrastructure. Overall, the evaluated systems are given "good" grades [9].

The administrative feasibility sub-criterion evaluates the efficiency of the entity that is enforcing the scheme. It is also the most crucial consideration in this criterion. The three schemes need a similar amount of work to implement their mechanisms. Conventional ETS relies on the relevant entities performing this work in an efficient and timely manner. The blockchain supported systems relies on the machines, smart devices, and the developed algorithms to implement the work. Hence, fewer entities and less effort are needed. For the proposed scheme, additional effort from experts is required to establish the carbon standards for the products. However, once the system is established, less human effort is needed to operate the ETS. Therefore, the proposed ETSs are evaluated with an average score of 6.5 for this sub-criterion. 
Finally, the financial feasibility assesses various costs involved during preparation, administration, reporting, and operation. The blockchain supported schemes have higher costs to establish the infrastructure, but the process automation reduces the costs of administration, reporting and operation. Due to the pros and cons of the blockchain supported ETS, the overall performance is considered at the "good" level.

\subsection{Summary of Evaluation}

The scores of the compared systems are summarized in Table 3. It could be seen that the overall performance of the blockchain supported systems are better than the conventional ETS. The conventional ETS in FAMI is scored slightly lower than that a generalized ETS. Compared with the conventional ETS, the BCELF offers significant improvements, especially in terms of the environmental performance and the political acceptability. The presented result clearly demonstrates the advantages of the proposed scheme over the conventional scheme.

Table 3. Grade of the multi-criteria evaluation of the conventional ETS, the BCRB, the conventional ETS in FAMI, and the BCELF.

\begin{tabular}{|c|c|c|c|c|c|c|c|}
\hline Criteria & $\begin{array}{c}\text { Weight } \\
\text { Coefficient [72] }\end{array}$ & $\begin{array}{c}\text { Conventional } \\
\text { ETS }\end{array}$ & $\begin{array}{c}\text { BCRB } \\
\text { [9] }\end{array}$ & $\begin{array}{l}\text { Conventional } \\
\text { ETS in FAMI }\end{array}$ & \multicolumn{3}{|c|}{ BCELF } \\
\hline 1. Environmental performance & 0.168 & 1.18 & $\begin{array}{l}\text { Ave. } \\
1.37\end{array}$ & 0.84 & $\begin{array}{l}\text { Min. } \\
1.38\end{array}$ & $\begin{array}{l}\text { Max. } \\
1.53\end{array}$ & $\begin{array}{l}\text { Ave. } \\
1.45\end{array}$ \\
\hline 1.1. Direct contribution to GHG emission reduction & 0.833 & 7 & 8.15 & 5 & 8.2 & 9.1 & 8.65 \\
\hline 1.2. Indirect environmental effects & 0.167 & 7 & 8.15 & 5 & 8.2 & 9.1 & 8.65 \\
\hline 2. Political acceptability & 0.738 & 4.86 & 5.15 & 4.92 & 5.45 & 5.75 & 5.60 \\
\hline 2.1. Static (cost) efficiency & 0.474 & 7 & 6.90 & 7 & 7.2 & 7.5 & 7.35 \\
\hline 2.2. Dynamic (cost) efficiency & 0.183 & 6 & 8.75 & 6 & 8.6 & 9 & 8.80 \\
\hline 2.3. Competitiveness & 0.085 & 5 & 4.25 & 6 & 4.5 & 5 & 4.75 \\
\hline 2.4. Equity & 0.175 & 7 & 6.25 & 7 & 8 & 8.5 & 8.25 \\
\hline 2.5. Flexibility & 0.051 & 7 & 6.85 & 7 & 6.8 & 7.2 & 7.00 \\
\hline 2.6. Stringency for non-compliance & 0.032 & 5 & 9.25 & 5 & 8.5 & 9.5 & 9.00 \\
\hline 3. Feasibility of implementation & 0.094 & 0.66 & 0.65 & 0.66 & 0.60 & 0.65 & 0.63 \\
\hline 3.1. Implementation network capacity & 0.309 & 7 & 6.95 & 7 & 6.7 & 7 & 6.85 \\
\hline 3.2. Administrative feasibility & 0.581 & 7 & 7.00 & 7 & 6.2 & 6.8 & 6.50 \\
\hline \multirow[t]{2}{*}{ 3.3. Financial feasibility } & 0.110 & 7 & 6.85 & 7 & 6.8 & 7.3 & 7.05 \\
\hline & Weighted total & 6.69 & 7.17 & 6.42 & 7.43 & 7.92 & 7.68 \\
\hline
\end{tabular}

\section{Discussions}

Although ETS has been implemented in many nations, very few ETSs are currently focused on the FAMI, which is a traditional yet highly polluting industry sector. The proposed BCELF attempts to address the issue. However, the presented study is just the beginning of a series of work to reduce the carbon emission in FAMI. This section presents a brief discussion about the challenges and the limits of the BCELF.

First, with the "lead by the government, administrated by everyone" scheme, the government authorities should find capital to facilitate the development and implementation of the system. For the BCELF, money and effort are needed to build the blockchain, operate the ETS, designate the auditors, and establish the carbon emission evaluation standards, etc.

Second, BCELF cannot fully solve all problems of existing ETSs [40,42,76]. For example, the merge of ETS across different industries remains a challenge due to different standards and the carbon emission allowances. In addition, for many products, there are no similar or compatible products, which make it hard to establish the standards. Moreover, as the carbon emission is evaluated at the product level, the boundary of the firms and the boundary of the products need to be carefully defined to avoid incompatibility issues during the implementation [77].

\section{Conclusions}

Environmental sustainability is receiving more and more public awareness all around the globe. Such trend leads to a new sustainability mindset, which facilitates newer environmental management systems. The study first provides a quick review of the blockchain and its existing applications to 
improve sustainability. Then, a blockchain enhanced ETS solution (BCELF) customized for Industry 4.0 integration is proposed for the fashion apparel manufacturing industry (FAMI). A novel emission link system is proposed at the product level to reduce the emissions for all the key steps of clothing making. The enhanced ETS system includes not only the authority, the auditors, the firms but also the individuals. Supported by blockchain and the smart measurement devices, the carbon emission could be easily measured and recorded with less human labor.

The provided system offers a systematic and feasible evaluation if the carbon emission for FAMI. To better demonstrate the carbon emission process, a detailed case study is presented in the paper for a prototype product. Carbon emissions for FAMI are evaluated over four aspects, i.e., supply, energy, labor, and wastes. Furthermore, the mathematical expressions to consider the uncertainties are provided.

The proposed system framework is a reliable approach to reduce carbon emissions for FAMI. First, the blockchain provides automation, transparency and immutability to the system. Second, as the framework is specially designed for FAMI, the solutions are highly compatible with the industry sector. Subsequently, a multi-criteria evaluation is performed for the BCELF. The results also prove that the BCELF offers significant improvements compared with the conventional ETS in FAMI, especially in terms of the environmental performance and the political acceptability.

Finally, the BCELF got some limitations, two challenges currently perceived are briefly provided in the study. Moreover, the future research topics could be: First, the carbon emission evaluation functions for various supplies, energy, wastes, and labors need to be carefully derived and analyzed. The carbon emission standards should be established impartially. These studies are important to the BCELF before its implementation. Second, the current individual engagement is relatively weak in the presented framework. Ideas to further promote the environmental awareness of the individuals based on the provided system are desired. In addition, the social/cultural and economic effects of the presented framework could be further studied in detail.

Acknowledgments: The authors gratefully acknowledge the support by Shanghai style fashion design \& value creation collaborative innovation center (ZX201311000031) for this research.

Author Contributions: Bailu Fu, Zhan Shu and Xiaogang Liu designed the study and wrote the paper together.

Conflicts of Interest: The authors declare no conflict of interest.

\section{References}

1. Lasi, H.; Kemper, H.G.; Fettke, P.; Feld, T.; Hoffmann, M. Industry 4.0. Bus. Inf. Syst. Eng. 2014, 6, $239-242$. [CrossRef]

2. Liao, Y.; Deschamps, F.; Loures, E.F.R.; Ramos, L.F.P. Past, present and future of Industry 4.0-a systematic literature review and research agenda proposal. Int. J. Prod. Res. 2017, 55, 3609-3629. [CrossRef]

3. Roblek, V.; Meško, M.; Krapež, A. A complex view of industry 4.0. SAGE Open 2016, 6, 1-11. [CrossRef]

4. Long, F.; Zeiler, P.; Bertsche, B. Modelling the production systems in industry 4.0 and their availability with high-level petri nets. In IFAC-PapersOnLine, Proceedings of the 8th IFAC Conference on Manufacturing Modelling, Management and Control (MIM), Troyes, France, 28-30 June 2016; Elsevier Science B.V.: Amsterdam, The Netherlands, 2016.

5. Gruber, F.E. Industry 4.0: A best practice project of the automotive industry. In Digital Product and Process Development Systems, Proceedings of the IFIP TC 5 International Conference on Project Research on Leading-Edge Applications and Methods for Applied Information Technology, Dresden, Germany, 10-11 October 2013; Springer: Berlin, Germany, 2013.

6. Wan, J.; Tang, S.; Shu, Z.; Li, D.; Wang, S.; Imran, M.; Vasilakos, A. Software-defined industrial internet of things in the context of Industry 4.0. IEEE Sens. J. 2016, 16, 7373-7380. [CrossRef]

7. Lu, Y. Industry 4.0: A survey on technologies, applications and open research issues. J. Ind. Inf. Integr. 2017, 6, 1-10. [CrossRef]

8. Sikorski, J.J.; Haughton, J.; Kraft, M. Blockchain technology in the chemical industry: Machine-to-machine electricity market. Appl. Energy 2017, 195, 234-246. [CrossRef] 
9. Khaqqi, K.N.; Sikorski, J.J.; Hadinoto, K.; Kraft, M. Incorporating seller/buyer reputation-based system in blockchain-enabled emission trading application. Appl. Energy 2018, 209, 8-19. [CrossRef]

10. Global Coin Report. Understanding the Core Concepts Behind QTUM. Available online: https:// globalcoinreport.com/understanding-core-concepts-behind-qtum (accessed on 11 February 2018).

11. Energy Blockchain Labs. Available online: http://www.energy-blockchain.com/EnHome (accessed on 11 February 2018).

12. EP-Chain. Available online: http:/ / www.ep-chain.org (accessed on 6 February 2018). (In Chinese)

13. Martin, M. Creating Sustainable Apparel Value Chains. Impact Economy. 2013, pp. 1-41. Available online: https:/ / aicprogram.com/reports/IE_PRIMER_DECEMBER2013_EN.pdf (accessed on 5 March 2018).

14. Chen, H.-L.; Burns, L.D. Environmental analysis of textile products. Cloth. Text. Res. J. 2006, 24, $248-261$. [CrossRef]

15. Zaffalon, V. Climate change, carbon mitigation and textiles. Text. World 2010, 160, 34-35.

16. Nakamoto, S. Bitcoin: A peer-to-peer electronic cash system. Available online: https://bitcoin.org/bitcoin. pdf (accessed on 31 January 2018).

17. Swan, M. Blockchain: Blueprint for a New Economy; O’Reilly Media Inc.: Sebastopol, CA, USA, 2015; p. xi, ISBN 9781491920497.

18. Bhargavan, K.; Delignat-Lavaud, A.; Fournet, C.; Gollamudi, A.; Gonthier, G.; Kobeissi, N.; Kulatova, N.; Rastogi, A.; Sibut-Pinote, T.; Swamy, N. Formal verification of smart contracts short paper. In Proceedings of the 2016 ACM Workshop on Programming Languages and Analysis for Security (PLAS'16), 11th ACM Workshop on Programming Languages and Analysis for Security (PLAS), Vienna, Austria, 24 October 2016; Association Computing Machinery: New York, NY, USA, 2016.

19. Jakobsson, M.; Juels, A. Proofs of work and bread pudding protocols. In Secure Information Networks: Communications and Multimedia Security, Proceedings of the IFIP TC6/TC11 Joint Working Conference on Communication and Multimedia Security (CMS 99), Leuven, Belgium, 20-21 September 1999; Kluwer Academic Publishers: Norwell, MA, USA, 1999.

20. Boneh, D.; Di Crescenzo, G.; Ostrovsky, R.; Persiano, G. Public key encryption with keyword search. In Advances in Cryptology-Eurocrypt 2004, Proceedings of the 23rd Annual Eurocrypt Conference, Interlaken, Switzerland, 2-6 May 2004; Springer: Berlin, Germany, 2004.

21. Aitzhan, N.Z.; Svetinovic, D. Security and privacy in decentralized energy trading through multi-signatures, blockchain and anonymous messaging streams. IEEE Trans. Dependable Secur. Comput. 2016, 1-14. [CrossRef]

22. Pop, C.; Cioara, T.; Antal, M.; Anghel, I.; Salomie, I.; Bertoncini, M. Blockchain based decentralized management of demand response programs in smart energy grids. Sensors 2018, 18. [CrossRef] [PubMed]

23. Aune, R.T.; Krellenstein, A.; O'Hara, M.; Slama, O. Footprints on a blockchain: Trading and information leakage in distributed ledgers. J. Trading 2017, 12, 5-13. [CrossRef]

24. Liu, W.; Wang, T.; Liu, Y.; Sun, C.; Zeng, M. Research on the technology and economic calculation model of power transmission line considering environmental benefits. In AIP Conference Proceedings, Proceedings of the International Conference on Materials Science, Resource and Environmental Engineering (MSREE), Xian, China, 10-11 December 2016; American Institute of Physics: Melville, NY, USA, 2017.

25. Hwang, J.; Choi, M.I.; Lee, T.; Jeon, S.; Kim, S.; Park, S.; Park, S. Energy prosumer business model using blockchain system to ensure transparency and safety. In Power And Energy Systems Engineering (CPESE 2017), Proceedings of the 4th International Conference on Power and Energy Systems Engineering (CPESE), Berlin, Germany, 25-29 September 2017; Elsevier Science B.V.: Amsterdam, The Netherlands, 2017.

26. Mylrea, M.; Gourisetti, S.N.G. Blockchain for smart grid resilience: Exchanging distributed energy at speed, scale and security. In Proceedings of the Resilience Week (RWS) 2017, Wilmington, DE, USA, 18-22 September 2017; IEEE-Inst Electrical Electronics Engineers Inc.: Piscataway, NJ, USA, 2017.

27. Hou, J.; Wang, H.; Liu, P. Applying the blockchain technology to promote the development of distributed photovoltaic in China. Int. J. Energy Res. 2018, 1-12. [CrossRef]

28. Exergy. Available online: https://lo3energy.com (accessed on 27 February 2018).

29. Mengelkamp, E.; Garttner, J.; Rock, K.; Kessler, S.; Orsini, L.; Weinhardt, C. Designing microgrid energy markets a case study: The brooklyn microgrid. Appl. Energy 2018, 210, 870-880. [CrossRef]

30. SolarCoin. Available online: https:/ / solarcoin.org/en/front-page (accessed on 8 March 2018).

31. Grid Singularity. Available online: http://gridsingularity.com (accessed on 1 March 2018).

32. Grid+. Available online: https://gridplus.io (accessed on 1 March 2018). 
33. Zhou, Y.; Wu, J.; Long, C.; Cheng, M.; Zhang, C. Performance evaluation of peer-to-peer energy sharing models. Energy Procedia 2017, 143, 817-822. [CrossRef]

34. PONTON-The Enerchain Project. Available online: https://enerchain.ponton.de (accessed on 2 March 2018).

35. Share\&Charge. Available online: https://shareandcharge.com (accessed on 17 March 2018).

36. Wood, G. ETHEREUM: A Secure Decentralised Generalized Transaction Ledger. Available online: http: / / gavwood.com/paper.pdf (accessed on 18 July 2017).

37. Oxygen Initiative. Available online: https:/ / oxygeninitiative.com (accessed on 17 March 2018).

38. Starting from the Micro Power Grid, Energo Tries to Build a Decentralized Autonomous Energy Transaction System Using Blockchain Technologies. Available online: http:/ /www.8btc.com/energo-labs-blockchain (accessed on 25 January 2018). (In Chinese)

39. Breidenich, C.; Magraw, D.; Rowley, A.; Rubin, J.W. Kyoto protocol to the United Nations framework convention on climate change. Am. J. Int. Law 1998, 92, 315-331. [CrossRef]

40. Bond, P. Emissions trading, new enclosures and eco-social contestation. Antipode 2011, 44, 684-701. [CrossRef]

41. Schneider, L.; Kollmuss, A.; Lazarus, M. Addressing the risk of double counting emission reductions under the UNFCCC. Climatic Change 2015, 131, 473-486. [CrossRef]

42. Segura, S.; Ferruz, L.; Gargallo, P.; Salvador, M. Environmental versus economic performance in the EU ETS from the point of view of policy makers: A statistical analysis based on copulas. J. Clean. Prod. 2018, 176, 1111-1132. [CrossRef]

43. Ellerman, A.D.; Buchner, B.K. The European Union Emissions Trading Scheme: Origins, allocation, and early results. Rev. Environ. Econ. Policy 2007, 1, 66-87. [CrossRef]

44. CleanMetrics. Carbon Footprint Analysis for Clothing and Accessories. Available online: http://www. cleanmetrics.com/html/clothing_carbon_footprints.htm (accessed on 5 April 2017).

45. World Wildlife Fund. Cutting Cotton Carbon Emissions: Findings from Warangal, India. WWF Report. 2013. Available online: http:/ /www.indiaenvironmentportal.org.in/files/file/cotton_carbon_emission.pdf (accessed on 5 March 2018).

46. Giacomin, A.M.; Garcia, J.B.; Zonatti, W.F.; Silva-Santos, M.C.; Laktim, M.C.; Baruque-Ramos, J. Silk industry and carbon footprint mitigation. In IOP Conference Series-Materials Science and Engineering, Proceedings of the 17th World Textile Conference of the Association-of-Universities-for-Textiles (AUTEX)—Shaping the Future of Textiles, Corfu, Greece, 29-31 May 2017; IOP Publishing Ltd.: Bristol, UK, 2017.

47. Perry, P. Exploring the influence of national cultural context on CSR implementation. J. Fash. Mark. Manag. 2012, 16, 141-160. [CrossRef]

48. de Brito, M.P.; Carbone, V.; Blanquart, C.M. Towards a sustainable fashion retail supply chain in Europe: Organisation and performance. Int. J. Prod. Econ. 2008, 114, 534-553. [CrossRef]

49. Weber, C.L.; Matthews, H.S. Quantifying the global and distributional aspects of American household carbon footprint. Ecol. Econ. 2008, 66, 379-391. [CrossRef]

50. Bocken, N.M.P.; Allwood, J.M. Strategies to reduce the carbon footprint of consumer goods by influencing stakeholders. J. Clean. Prod. 2012, 35, 118-129. [CrossRef]

51. Choi, T.-M. Local sourcing and fashion quick response system: The impacts of carbon footprint tax. Transp. Res. Part E Logist. Transp. Rev. 2013, 55, 43-54. [CrossRef]

52. Waste and Resources Action Programme (WRAP) 2012 Final Report-A Carbon Footprint for UK Clothing and Opportunities for Savings. Available online: http:/ / www.wrapni.org.uk/sites/files/wrap/Appendix\% 20IV\%20-\%20Carbon\%20footprint\%20report.pdf (accessed on 5 February 2018).

53. Congressional Budget Office (CBO). Policy Options for Reducing CO2 Emissions: A CBO Study. Available online: http:/ / www.cbo.gov/sites/default/ files/02-12-carbon.pdf (accessed on 10 February 2018).

54. Chen, X.; Benjaafar, S.; Elomri, A. The carbon-constrained EOQ. Oper. Res. Lett. 2012, 41, 172-179. [CrossRef]

55. Song, J.; Leng, M. Analysis of the single-period problem under carbon emissions policies. In Handbook of Newsvendor Problems; Choi, T.-M., Ed.; Springer: New York, NY, USA, 2012; Volume 176, pp. 297-313, ISBN 9781461435990.

56. He, L.; Zhao, D.; Xia, L. Game theoretic analysis of carbon emission abatement in fashion supply chains considering vertical incentives and channel structures. Sustainability 2015, 7, 4280-4309. [CrossRef]

57. Adidas Sustainability Reports. Available online: https://www.adidas-group.com/en/sustainability/ reporting/sustainability-reports (accessed on 10 March 2018). 
58. H\&M Sustainability Report. Available online: http:/ / sustainability.hm.com (accessed on 10 March 2018).

59. Marks and Spencer's Plan A. Available online: https://corporate.marksandspencer.com/plan-a (accessed on 11 February 2018).

60. Nike Sustainable Nusiness Report. Available online: https://about.nike.com/pages/sustainable-innovation (accessed on 10 March 2018).

61. Lin, Y.-P.; Petway, J.R.; Anthony, J.; Mukhtar, H.; Liao, S.-W.; Chou, C.-F.; Ho, Y.-F. Blockchain: The evolutionary next step for ICT e-agriculture. Environments 2017, 4. [CrossRef]

62. Partnership for Market Readiness (PMR); International Carbon Action Partnership (ICAP). Emission Trading in Practice: A Handbook on Design and Implementation; World Bank: Washington, DC, USA, 2016.

63. Choudhury, A.K.R. Environmental impacts of the textile industry and its assessment through life cycle assessment. In Roadmap to Sustainable Textiles and Clothing: Environmental and Social Aspects of Textiles and Clothing Supply Chain; Muthu, S.S., Ed.; Springer: Singapore, 2014; pp. 1-39, ISBN 978-981-287-109-1.

64. Shen, B.; Li, Q.; Dong, C.; Perry, P. Sustainability issues in textile and apparel supply chains. Sustainability 2017, 9, 1592. [CrossRef]

65. Karthik, T.; Gopalakrishnan, D. Environmental analysis of textile value chain: An overview. In Roadmap to Sustainable Textiles and Clothing: Environmental and Social Aspects of Textiles and Clothing Supply Chain; Muthu, S.S., Ed.; Springer: Singapore, 2014; pp. 153-188, ISBN 978-981-287-109-1.

66. Griffths-Sattenspiel, B.; Wilson, W. The Carbon Footprint of Water; River Network: Portland, OR, USA, 2009; pp. 21-24.

67. Zhou, Y.; Zhang, B.; Wang, H.; Bi, J. Drops of energy: Conserving urban water to reduce greenhouse gas emissions. Environ. Sci. Technol. 2013, 47, 10753-10761. [CrossRef] [PubMed]

68. Duan, C.; Chen, B. Energy-water-carbon nexus at urban scale. In Energy Procedia, Proceedings of the Applied Energy Symposium and Summit-Low-Carbon Cities and Urban Energy Systems (CUE), Jinan, China, 13-15 June 2016; Elsevier Science B.V.: Amsterdam, The Netherlands, 2016.

69. Bao, Z.; Sun, S.; Sun, D. Characteristics of direct CO2 emissions in four full-scale wastewater treatment plants. Desalination Water Treat. 2014, 54, 1070-1079. [CrossRef]

70. Yu, L.; Wang, L.; Chen, Y. Calculation model of carbon emission in garment production processes. J. Text. Res. 2016, 37, 156-159. (In Chinese)

71. Kirchner, J. Data Analysis Toolkit \#5: Uncertainty Analysis and Error Propagation; Berkeley Seismology Laboratory, University of California. 2001. Available online: http:/ / seismo.berkeley.edu/ kirchner/eps_ 120/Toolkits/Toolkit_05.pdf (accessed on 17 March 2018).

72. Konidari, P.; Mavrakis, D. A multi-criteria evaluation method for climate change mitigation policy instruments. Energy Policy 2007, 35, 6235-6257. [CrossRef]

73. Clò, S.; Battles, S.; Zoppoli, P. Policy options to improve the effectiveness of the EU emissions trading system: A multi-criteria analysis. Energy Policy 2013, 57, 477-490. [CrossRef]

74. Khoshnava, S.M.; Rostami, R.; Valipour, A.; Ismail, M.; Rahmat, A.R. Rank of green building material criteria based on the three pillars of sustainability using the hybrid multi criteria decision making method. J. Clean. Prod. 2018, 173, 82-99. [CrossRef]

75. Venmans, F.M.J. The effect of allocation above emissions and price uncertainty on abatement investments under the EU ETS. J. Clean. Prod. 2016, 126, 595-606. [CrossRef]

76. Kopsch, F. Aviation and the EU Emissions Trading Scheme-Lessons learned from previous emissions trading schemes. Energy Policy 2012, 49, 770-773. [CrossRef]

77. Some Ideas on the Allocation of Carbon Emission Allowance. Available online: http:/ /www.tanjiaoyi.com/ article-22082-1.html (accessed on 17 March 2018). (In Chinese)

(C) 2018 by the authors. Licensee MDPI, Basel, Switzerland. This article is an open access article distributed under the terms and conditions of the Creative Commons Attribution (CC BY) license (http:/ / creativecommons.org/licenses/by/4.0/). 